\title{
RELATION BETWEEN BCDS AND BLUE LSBGS
}

\author{
NILS BERGVALL, GÖRAN ÖSTLIN, ARNAUD PHARASYN AND JARI RÖNNBACK \\ Astronomical Observatory, Box 515, S-75120 Uppsala, Sweden
}

AND

JOSEFA MASEGOSA

Instituto de Astrofísica de Andalucí, P.O. Box 3004, 18080 Granada, Spain

\section{Introduction}

Two important questions are connected to the evolutionary history of blue compact dwarf galaxies (BCDs): the triggering mechanism of the starburst and the properties of the precursor(s) and successor. The halos of many BCDs show red colours indicating that the host galaxy is in fact of early type. But where did the gas come from? Was it there from the start in a quiescent mode or was it fed by a merger with an intergalactic gas cloud or a gas-rich dwarf? Is there a general answer to this question or are there different families of BCDs?

The BCDs generating most of the interest are those with low chemical abundances, typically $10 \%$ solar. The only plausible precursors in this case are metal-poor dIs, LSBGs or pure HI clouds (e.g. Thuan \& Seitzer 1979, ApJ 231, 680). In a comparison between LSBGs and BCDs Taylor et al. (e.g. 1997, ApJ 480, 524; 1996, ApJS 107, 143) found an excess of HI rich companions in the environments of BCDs which they interpret as a support of tidal triggering. We have studied a few massive $\left(\sim 10^{9} \mathcal{M}_{\odot}\right)$ metal-poor blue compact galaxies (BCGs). These are too bright to be classified as dwarfs but define an extension of the BCD family. For simplicity we will in the following use the common abbrevation BCD. In a search for possible precursors we have studied a sample of blue LSBGs with metallicities similar to BCDs (5-15\%).

\section{HI masses and chemical abundances}

Fig. 1 shows the $\mathcal{M}_{\mathrm{HI}} / \mathrm{M}_{B}$ relation for BCDs (Thuan and Martin 1981, ApJ 247,823) applying the restriction B-V $\leq 0.6$; LSBGs (from McGaugh et al. 1997, ApJ 481, 689) with the restriction $\mu_{B, 0} \geq 22.0$ and $\mathrm{B}-\mathrm{V} \leq 0.6$; and blue LSBGs (Bergvall et al., in prep.). Least-square fits to the data show that the regression lines for the two types of galaxies run nearly parallel. If we induce starformation in an LSBG to produce a BCD, the shift indicates an increase in luminosity of 1.5 mag. Fig $1 b$ shows the metallicity-luminosity diagram for nearby dwarf galaxies (Skillman et al. 1989, ApJ 347, 875), blue LSBGs (Rönnback and Bergvall 1994 A\&AS 108, 193; 1995, A\&A 302, 353) and four luminous BCDs with low metallicities (Bergvall et al., in prep.). The positions of the two most popular young galaxy candidates, IZw 18 and SBS 0335-052 are also indicated. These have $\mathcal{M}_{\mathrm{HI}} / \mathrm{L}_{B}$ similar to LSBGs whereas the more massive ones follow the BCD relation in Fig 1a. For the dIs we have used the M--Z relationship from Skillman et al. (1989). A similar fit to the LSBGs (dotted line), assuming that its slope is the same as for the dwarfs, gives a significant shift towards higher luminosities and/or lower Z. If we now, on the basis of the HI data assume that we, by turning on a burst in the LSBG, increase its luminosity by 1.5 mag and at the same time demand that the metallicity does not increase we hit the next line running through the BCDs (dashed line). Thus, the combined HI data and metallicities support a relationship between BCDs and blue LSBGs. If we want to use the dIs in the diagram to fabricate the low metallicity BCDs we have to increase the luminosity by 3 mags. This makes it much more difficult since: 1 ) the star formation efficiency has to be nearly $100 \%$ and 2) the resulting $\mathcal{M}_{\mathrm{HI}} / \mathrm{L}_{B}$ will be much higher than the observed value for BCDs (the $\mathcal{M}_{\mathrm{HI}} / \mathrm{L}_{B}$ for dIs is lower than for LSBGs). 

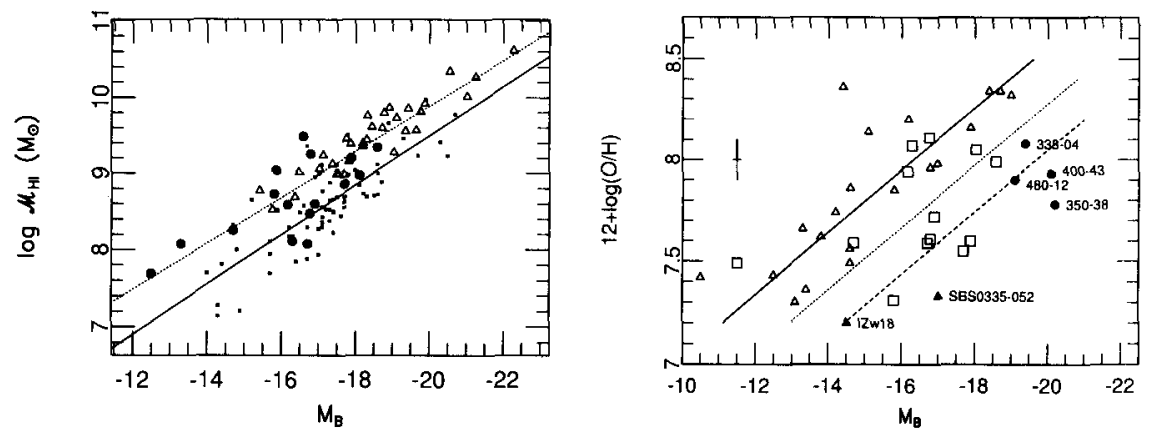

Figure 1. a) The $\mathcal{M}_{\mathrm{H}} / \mathrm{M}_{B}$ relationship for blue LSBGs (subsample of McGaugh et al, triangles) blue LSBGs (circles) and BCDs (Thuan \& Martin; dots). The regression lines for the samples are indicated. b) The metallicity-luminosity relationship for dIs (Skillman et al., triangles), blue LSBGs (Rönnback \& Bergvall, squares), the BCDs ESO 338-04, $350-38,400-43$ and $480-12$ (Bergvall et al., filled circles), I Zw 18 and SBS 0335-052 (filled triangles)

\section{Photometric properties}

Scale lengths and central surface brightnesses can often put useful constraints on the evolution of different types of galaxies. Disk type LSBGs are particularly simple systems in this respect. The central surface brightness of massive BCDs is $\approx 4$ magnitudes brighter than a LSBG while the required increase in total luminosity according to the above is 1.5 mag. We thus have to focus the starburst towards the centre and steepen the luminosity profile. At the same HI mass the BCDs thus should have a shorter scale length, in accordance with observations. In such galaxies one would hope to detect the precursor disk at large radii and faint levels. Exponential profiles have been detected in several cases (e.g. Papaderos et al. 1996, A\&AS 120,207) but the derived central surface brightness of this component is significanly brighter than the mean value for LSBGs $\left(\mu_{B, 0} \approx 23-23.5\right)$. Normally the measured profiles stop at $\mu_{B} \approx 26-27$. From a study of 'our' four luminous BCDs, however, we have found that at an even fainter level, $\mu_{B} \approx 28-29$, the profile will continue to flatten out. When combining near-IR colours with optical we find that in two cases, ESO 400-G43 and ESO 350-IG38, the outer profile has colours of an early type galaxy while in the other two cases it may very well be an LSBG disk. To make further constraints, we have made comparisons between their observed broadband colours and those predicted by our spectral evolutionary models (SEMs). We find that the ages of the 'dE' (=dSph) component in the first two cases is of the order of $10 \mathrm{Gyr}$. In the other two cases the colours are similar to those of blue LSBGs, indicating a continuous star formation history.

Strong support for the presence of a dE host comes from recent HST observations of ESO 338IG04 (Östlin et al., in prep.), in which we have detected a system of globular clusters (GCs). Our photometry is sufficiently good so that we can use the SEMs to derive age estimates. The results point at three distinct age groups of the GCs, one old, one of an age of $\sim 2 \mathrm{Gyr}$ and one young. From the luminosity function of the old GC population, corrected for incompleteness, we can derive the total number of old GCs. Comparing this with the luminosity of the underlying galaxy, we find it to be consistent only with a very $\mathrm{GC}$ rich $\mathrm{dE}$, with $\mathrm{M}_{B} \sim-18$.

\section{Central kinematics}

We (Östlin et al., in prep.) recently used Fabry-Perot observations to study the H $\alpha$ velocity field in a sample of BCDs and their companions, including the ones discussed above. The general result is that the velocity field in the companions is quite ordered. The main components, however, show a higher complexity. The most likely interpretation is that mergers are frequent and probably responsible for the starburst activity. From these results we draw the conclusion that an attractive scenario for the ignition of a BCD is that a merger is taking place - involving an LSBG and sometimes a $\mathrm{dE}$ (possibly containing a gas disk). 